\title{
Mapping $\alpha_{2}$ Adrenoceptors of the Human Brain with ${ }^{11}$ C-Yohimbine
}

\author{
Adjmal Nahimi ${ }^{1}$, Steen Jakobsen ${ }^{1}$, Ole L. Munk ${ }^{1}$, Kim Vang ${ }^{1}$, Jenny A. Phan ${ }^{1}$, Anders Rodell ${ }^{1}$, and Albert Gjedde ${ }^{1-4}$ \\ ${ }^{1}$ Department of Nuclear Medicine and PET Centre, Aarhus University Hospitals, Aarhus, Denmark; ${ }^{2}$ Department of Neuroscience \\ and Pharmacology, University of Copenhagen, Copenhagen, Denmark; ${ }^{3}$ Department of Radiology and Radiological Science, Johns \\ Hopkins University, Baltimore, Maryland; and ${ }^{4}$ Department of Neurology, McGill University, Montreal, Quebec, Canada
}

\begin{abstract}
A previous study from this laboratory suggested that ${ }^{11} \mathrm{C}$-yohimbine, a selective $a_{2}$-adrenoceptor antagonist, is an appropriate ligand for PET of $a_{2}$ adrenoceptors that passes readily from blood to brain tissue in pigs but not in rodents. To test usefulness in humans, we determined blood-brain clearances, volumes of distribution, and receptor availability by means of PET with ${ }^{11} \mathrm{C}$-yohimbine in healthy male adults. Methods: We recorded the distribution of ${ }^{11} \mathrm{C}$-yohimbine with 90-min dynamic PET and sampled arterial blood to measure intact ${ }^{11} \mathrm{C}$-yohimbine in plasma. For analysis, we coregistered PET images to individual MR images and automatically identified 27 volumes of interest. We used 1-tissue-compartment graphical analysis with 6 linearized solutions of the fundamental binding equation, with the metabolite-corrected arterial plasma curves as input function, to estimate the kinetic parameters of ${ }^{11} \mathrm{C}$-yohimbine. With the lowest steady-state distribution volume $\left(V_{T}\right)$, determined in the corpus callosum, we calculated the binding potential (receptor availability) of the radioligand in other regions. Results: The linear regressions yielded similar estimates of the kinetic parameters. The cortical values of $V_{T}$ ranged from $0.82 \mathrm{~mL} \mathrm{~cm}^{-3}$ in the right frontal cortex to 0.46 $\mathrm{mL} \mathrm{cm}{ }^{-3}$ in the corpus callosum, with intermediate $V_{T}$ values in subcortical structures. Binding potentials averaged 0.6-0.8 in the cortex and $0.2-0.5$ in subcortical regions. Conclusion: The maps of ${ }^{11} \mathrm{C}$ yohimbine binding to $a_{2}$ adrenoceptors in human brain had the highest values in cortical areas and hippocampus, with moderate values in subcortical structures, as found also in vitro. The results confirm the usefulness of the tracer ${ }^{11} \mathrm{C}$-yohimbine for mapping $\mathrm{a}_{2}$ adrenoceptors in human brain in vivo.
\end{abstract}

Key Words: ${ }^{11} \mathrm{C}$-yohimbine; $\mathrm{a}_{2}$ adrenoceptors; positron emission tomography; brain; noradrenaline

J Nucl Med 2015; 56:392-398

DOI: $10.2967 /$ jnumed.114.145565

\section{A} presynaptically on noradrenergic neurons, where they regulate firing of noradrenergic neurons as well as noradrenaline synthesis and release (1). Three $\alpha_{2}$-adrenoceptor subtypes have been identified, including the cloned human $\alpha_{2}$ adrenoceptors $\alpha_{2}-\mathrm{C} 10, \alpha_{2}-\mathrm{C} 2$, and $\alpha_{2}-\mathrm{C} 4$, labeled according to the chromosomal localization of

Received Aug. 10, 2014; revision accepted Nov. 18, 2014.

For correspondence or reprints contact: Adjmal Nahimi, Department of Nuclear Medicine and PET Centre, Aarhus University Hospitals, Denmark, Noerrebrogade 44, Building 10G, 6th Floor, 8000 Aarhus C, Denmark. E-mail: anah@ki.au.dk

Published online Jan. 29, 2015.

COPYRIGHT (C) 2015 by the Society of Nuclear Medicine and Molecular Imaging, Inc. the genes (2-4). The three $\alpha_{2}$-adrenoceptor subtypes are known as $\alpha_{2 \mathrm{a}}, \alpha_{2 \mathrm{~b}}$, and $\alpha_{2 \mathrm{c}}$ in the pharmacologic nomenclature (5).

Dense expression of $\alpha_{2}$ adrenoceptors occurs in the cerebral cortex, hippocampal formation, hypothalamus, and locus coeruleus, with intermediate-to-moderate binding in subcortical structures and low expression in white matter $(6,7)$. Degeneration of the noradrenergic neurotransmitter system and specific loss of $\alpha_{2}$ adrenoceptors is implicated in neurodegenerative disorders (8). Imaging of $\alpha_{2}$ adrenoceptors therefore is essential to understanding the role of the noradrenergic system in these disorders. However, no PET radioligands selective for $\alpha_{2}$ adrenoceptors have passed successfully from preclinical evaluation to clinical usefulness $(9,10)$.

We recently developed ${ }^{11} \mathrm{C}$-yohimbine for use with PET. Yohimbine binds to all $\alpha_{2}$-adrenoceptor subtypes and has moderate affinity for the $5 \mathrm{HT}_{1 \mathrm{~A}}$ receptors as well (11). In porcine brain, ${ }^{11} \mathrm{C}$-yohimbine enters brain tissue readily and binds specifically in brain areas with known high densities of $\alpha_{2}$ adrenoceptors. In rat brain, ${ }^{11} \mathrm{C}$-yohimbine is excluded from entry into brain tissue by the action of permeability glycoprotein (12). In porcine brain, both unlabeled yohimbine and RX821002 (2-(2-methoxy-1,4-benzodioxan-2yl)-2-imidazoline), a highly selective $\alpha_{2}$-adrenoceptor antagonist, displace ${ }^{11} \mathrm{C}$-yohimbine binding (11). These results prompted us to test ${ }^{11} \mathrm{C}$-yohimbine as a radioligand for use in humans, particularly with respect to the ability of the tracer to readily cross the blood-brain barrier.

\section{MATERIALS AND METHODS}

\section{Subjects}

The Committee on Ethics of the Central Denmark Region approved this study, and all subjects provided written informed consent before participation in this study. None of the subjects had any neurologic or psychiatric disorder. We recruited 6 young male subjects with a mean age of 28.7 y (SEM, $\pm 4.1 \mathrm{y})$ and an age range of 24-35 y to participate in the study. Subject 6 was excluded from analysis after the PET session, as this subject received a dose of radioactivity almost one order of magnitude lower than the other subjects and showed the highest rate of metabolism of yohimbine in the circulation (corresponding to the CYP2D6 status given in Table 1), resulting in the almost complete disappearance of radioactivity from the circulation.

\section{Radiochemistry, Metabolites, and CYP2D6 Status}

The synthesis of ${ }^{11} \mathrm{C}$-yohimbine has been described in detail elsewhere (11). Briefly, cyclotron-produced ${ }^{11} \mathrm{C}$-carbon dioxide was converted to ${ }^{11} \mathrm{C}$-methyliodide and trapped in dimethylsulfoxide $(300 \mu \mathrm{L})$ containing $\mathrm{NaOH}(1 \mu \mathrm{L}, 3 \mathrm{M})$ and yohimbinic acid $(1 \mathrm{mg})$ in a $1-\mathrm{mL}$ vial. This mixture was heated at $80^{\circ} \mathrm{C}$ for $3 \mathrm{~min} .{ }^{11} \mathrm{C}$-yohimbine was purified by semipreparative high-performance liquid chromatography. The mobile phase, consisting of $75 \%$ aqueous $70 \mathrm{mM} \mathrm{Na}_{2} \mathrm{HPO}_{4}$ and $25 \%$ ethanol, was delivered at a rate of $5 \mathrm{~mL} / \mathrm{min}$ to a LUNA C18(2) 
TABLE 1

Injected Radioactivity Dose, Yohimbine Mass, and CYP2D6 Status

\begin{tabular}{lccc}
\hline Subject & MBq & Yohimbine $(\mathrm{nmol})$ & CYP2D6 \\
\hline 1 & 641 & 30.3 & $1 \times 2(\mathrm{~F})$ \\
2 & 441 & 4.6 & $4 \times 1(\mathrm{M})$ \\
3 & 635 & 7 & $1 \times 4(\mathrm{M})$ \\
4 & 715 & 21.4 & $4 \times 4(\mathrm{~N})$ \\
5 & 507 & 11.2 & $2 \times 5(\mathrm{M})$ \\
6 & 169 & 11 & $2 \times 2(\mathrm{~F})$
\end{tabular}

$\mathrm{F}, \mathrm{M}$, and $\mathrm{N}=$ fast, moderate, and nonmetabolizers, respectively, of ${ }^{11} \mathrm{C}$-yohimbine.

$250 \times 10 \mathrm{~mm}$ semipreparative column (Phenomenex) with online radio wave and ultraviolet-visible $(280 \mathrm{~nm})$ detection. The fraction containing ${ }^{11} \mathrm{C}$-yohimbine (retention time, $7-8 \mathrm{~min}$ ) was collected and diluted with $5 \mathrm{~mL}$ of sterile saline and filtered through a sterile $0.22-\mu \mathrm{m}$ filter to obtain a total of $10 \mathrm{~mL}$ of product solution. This procedure gave 1-2 $\mathrm{GBq}$ of ${ }^{11} \mathrm{C}$-yohimbine with radiochemical purity greater than $99 \%$ in a sterile formulation ready for injection. With regard to metabolites of ${ }^{11} \mathrm{C}$-yohimbine in circulation, the fraction of untransformed ${ }^{11} \mathrm{C}$-yohimbine was measured by radio-high-performance liquid chromatography in extracts of plasma from samples taken at 2, 5, 10, 20, 40, 60, and 90 min after injection. We also genotyped the cytochrome P450 (CYP) system with regard to the CYP2D6 isoform at the laboratory Filedelfia, Denmark.

Yohimbinic acid monohydrate, yohimbine hydrochloride, dimethylsulfoxide, and acetonitrile were purchased from Sigma Aldrich.

\section{Image Acquisition, Registration, and Segmentation}

Subjects reclined in the scanner, an ECAT High Resolution Research Tomograph (HRRT; CTI/Siemens), and the head was positioned and immobilized in a custom-built head holder. A 6-min transmission scan was obtained, and a 90-min dynamic PET scan consisting of 28 frames increasing in duration from $15 \mathrm{~s}$ in the beginning to $10 \mathrm{~min}$ at the end of the scan $(8 \times 15 \mathrm{~s}, 4 \times 30 \mathrm{~s}, 6 \times 60 \mathrm{~s}$, $4 \times 300 \mathrm{~s}, 6 \times 600 \mathrm{~s}$ ) was recorded in 3 -dimensional mode on administration of ${ }^{11} \mathrm{C}$-yohimbine. An ordered-subsets expectation maximization 3-dimensional ordinary Poisson algorithm including point spread function modeling, with 10 iterations and 16 subsets, was used for reconstruction, resulting in $256 \times 256 \times 207$ images at a resolution of $1.8 \mathrm{~mm}$ in full width at half maximum. Blood samples were collected at 5-s intervals in the beginning increasing to intervals of $10 \mathrm{~min}$ at the end of the scan. The subjects underwent T1-weighted MR imaging. We used PMOD software, version 3.5, and the module PNEURO (PMOD Technologies Ltd.) for model-based image coregistration and segmentation of volumes of interest (VOIs). First, we averaged the dynamic images from frames $5-25$ to provide a sufficient anatomic PET image. We then coregistered the anatomic PET images to the individual anatomic MR images by a rigid coregistration method using a mutual-information algorithm. Individual MRI scans were spatially normalized to the Montreal Neurological Institute T1 template, and the transformation matrices were used to bring the dynamic PET scan into Montreal Neurological Institute space. VOIs were automatically outlined on the normalized MR images according to the Hammers maximum probability atlas, which is implemented in PMOD (13). The VOIs were intersected with a $50 \%$ gray matter probability mask and then applied to the spatially normalized dynamic PET scans to obtain time-activity curves for 27 selected VOIs (Table 2).

\section{Kinetic Analysis of ${ }^{11} \mathrm{C}$-Yohimbine Uptake and Distribution}

The kinetic parameters of ${ }^{11} \mathrm{C}$-yohimbine in the human brain were estimated with nonlinear 1- and 2-tissue-compartment models. There were no significant differences between estimates of ${ }^{11} \mathrm{C}$-yohimbine volumes of distribution or Akaike information criterion scores between these models. However, the nonlinear 2-tissue-compartment model produced nonphysiologic estimates of the kinetic parameters in some instances. For simplicity of analysis and illustration, we chose to compare the results of 6 linearizations of the 1-tissue-compartment model, as presented in the paragraphs below.

The distribution of a tracer as a function of time is governed by linear differential equations with constant transfer coefficients. In the case of a tracer that enters a brain tissue compartment in which bound and unbound tracer are in steady state, the distribution of the tracer is governed by the exchange between the circulation and the tissue compartment across the blood-brain and other potential barriers:

$$
\frac{d m_{1}}{d t}=V_{o} \frac{d c_{a}}{d t}
$$

Eq. 1

where $m_{1}(\mathrm{kBq} / \mathrm{mL})$ is the tracer quantity in the vascular compartment with the volume $V_{o}\left(\mathrm{~mL} / \mathrm{cm}^{3}\right)$ and the concentration $c_{a}(t)(\mathrm{kBq} / \mathrm{mL})$ as function of time, and

$$
\frac{d m_{2}}{d t}=K_{1} c_{a}-k_{2}^{\prime} m_{2}
$$

where $m_{2}(\mathrm{kBq} / \mathrm{mL})$ is the quantity of tracer in the tissue compartment, $K_{1}\left(\mathrm{~mL} / \mathrm{cm}^{3} / \mathrm{min}\right)$ is the unidirectional clearance of tracer from the circulation, and $k_{2}^{\prime}\left(\mathrm{min}^{-1}\right)$ is the rate constant of tracer efflux from the tissue compartment, equal to the ratio of the unidirectional clearance $K_{1}$ and the total steady-state volume of distribution $V_{T}\left(\mathrm{~mL} / \mathrm{cm}^{3}\right)$, here defined as

$$
k_{2}^{\prime}=\frac{k_{2}}{1+B P_{N D}}=\frac{K_{1}}{V_{N D}\left(1+B P_{N D}\right)}=\frac{K_{1}}{V_{T}} .
$$

where $k_{2}\left(\mathrm{~min}^{-1}\right)$ is the rate constant of efflux of dissolved (unbound) tracer from the tissue compartment, where the physical volume of distribution of tracer in solution is the volume of nondisplaceable tracer $V_{N D}\left(\mathrm{~mL} / \mathrm{cm}^{3}\right.$ ) (from which it cannot be displaced by blockade of the binding) and the binding potential, or steady-state ratio between bound and unbound tracer, is $B P_{N D}$.

When solved, the 2 differential equations yield

$$
m_{1}=V_{o} c_{a}
$$

and

$$
m_{2}=K_{1} \int_{0}^{T} c_{a} d t-k_{2}^{\prime} \int_{0}^{T} m_{2} d t
$$

from which the unknown quantity $m_{2}$ can be eliminated by substitution of $m_{2}=m-m_{1}$,

$$
m\left(1-\frac{m_{1}}{m}\right)=K_{1} \int_{0}^{T} c_{a} d t-k_{2}^{\prime} \int_{0}^{T}\left[m\left(1-\frac{m_{1}}{m}\right)\right] d t,
$$

which can be further reduced when the $m_{1} / m$ ratio approaches zero,

$$
m=K_{1} \int_{0}^{T} c_{a} d t-k_{2}^{\prime} \int_{0}^{T} m d t
$$

at a time that can be judged from the ratio as a function of time, 
TABLE 2

Kinetic Parameters of ${ }^{11} \mathrm{C}$-Yohimbine

\begin{tabular}{|c|c|c|c|c|}
\hline VOI & $V_{T}\left(\mathrm{~mL} / \mathrm{cm}^{3}\right)$ & $K_{1}\left(\mathrm{~mL} / \mathrm{cm}^{3} / \mathrm{min}\right)$ & $k_{2}^{\prime}\left(\min ^{-1}\right)$ & Binding potentia \\
\hline Amygdala, L & $0.55 \pm 0.11$ & $0.014 \pm 0.002$ & $0.026 \pm 0.002$ & $0.22 \pm 0.12$ \\
\hline Amygdala, $\mathrm{R}$ & $0.55 \pm 0.10$ & $0.014 \pm 0.002$ & $0.025 \pm 0.001$ & $0.23 \pm 0.07$ \\
\hline Caudate nucleus, L & $0.55 \pm 0.10$ & $0.016 \pm 0.003$ & $0.029 \pm 0.002$ & $0.24 \pm 0.13$ \\
\hline Caudate nucleus, $\mathrm{R}$ & $0.56 \pm 0.12$ & $0.016 \pm 0.003$ & $0.028 \pm 0.002$ & $0.23 \pm 0.11$ \\
\hline Cerebellum, L & $0.57 \pm 0.10$ & $0.019 \pm 0.003$ & $0.032 \pm 0.002$ & $0.28 \pm 0.11$ \\
\hline Cerebellum, R & $0.59 \pm 0.11$ & $0.020 \pm 0.003$ & $0.032 \pm 0.002$ & $0.32 \pm 0.11$ \\
\hline Corpus callosum & $0.46 \pm 0.09$ & $0.009 \pm 0.001$ & $0.019 \pm 0.003$ & - \\
\hline Frontal lobe, L & $0.80 \pm 0.16$ & $0.020 \pm 0.003$ & $0.023 \pm 0.001$ & $0.77 \pm 0.14$ \\
\hline Frontal lobe, $\mathrm{R}$ & $0.82 \pm 0.16$ & $0.020 \pm 0.003$ & $0.023 \pm 0.001$ & $0.81 \pm 0.13$ \\
\hline Gyrus cinguli, L & $0.80 \pm 0.16$ & $0.020 \pm 0.003$ & $0.025 \pm 0.001$ & $0.77 \pm 0.14$ \\
\hline Gyrus cinguli, R & $0.80 \pm 0.15$ & $0.020 \pm 0.003$ & $0.024 \pm 0.001$ & $0.77 \pm 0.14$ \\
\hline Hippocampus, L & $0.77 \pm 0.13$ & $0.016 \pm 0.003$ & $0.021 \pm 0.003$ & $0.73 \pm 0.14$ \\
\hline Hippocampus, R & $0.82 \pm 0.16$ & $0.014 \pm 0.002$ & $0.018 \pm 0.003$ & $0.82 \pm 0.21$ \\
\hline Insula, L & $0.72 \pm 0.14$ & $0.017 \pm 0.003$ & $0.023 \pm 0.001$ & $0.61 \pm 0.15$ \\
\hline Insula, R & $0.73 \pm 0.13$ & $0.017 \pm 0.003$ & $0.023 \pm 0.001$ & $0.62 \pm 0.12$ \\
\hline Occipital lobe, L & $0.78 \pm 0.15$ & $0.020 \pm 0.003$ & $0.024 \pm 0.001$ & $0.73 \pm 0.14$ \\
\hline Occipital lobe, R & $0.82 \pm 0.17$ & $0.020 \pm 0.004$ & $0.024 \pm 0.001$ & $0.80 \pm 0.12$ \\
\hline Parietal lobe, L & $0.78 \pm 0.15$ & $0.019 \pm 0.003$ & $0.023 \pm 0.001$ & $0.72 \pm 0.13$ \\
\hline Parietal lobe, $\mathrm{R}$ & $0.79 \pm 0.16$ & $0.019 \pm 0.003$ & $0.023 \pm 0.001$ & $0.75 \pm 0.11$ \\
\hline Putamen, L & $0.62 \pm 0.12$ & $0.021 \pm 0.004$ & $0.032 \pm 0.002$ & $0.40 \pm 0.15$ \\
\hline Putamen, R & $0.60 \pm 0.12$ & $0.020 \pm 0.004$ & $0.033 \pm 0.002$ & $0.33 \pm 0.12$ \\
\hline Temporal lobe, L & $0.75 \pm 0.13$ & $0.017 \pm 0.003$ & $0.022 \pm 0.001$ & $0.67 \pm 0.13$ \\
\hline Temporal lobe, $\mathrm{R}$ & $0.75 \pm 0.14$ & $0.017 \pm 0.003$ & $0.022 \pm 0.001$ & $0.66 \pm 0.12$ \\
\hline Thalamus, L & $0.62 \pm 0.12$ & $0.018 \pm 0.003$ & $0.029 \pm 0.002$ & $0.37 \pm 0.09$ \\
\hline Thalamus, R & $0.65 \pm 0.10$ & $0.019 \pm 0.004$ & $0.027 \pm 0.002$ & $0.46 \pm 0.11$ \\
\hline White matter, L & $0.59 \pm 0.11$ & $0.012 \pm 0.002$ & $0.020 \pm 0.001$ & $0.30 \pm 0.10$ \\
\hline White matter, $\mathrm{R}$ & $0.59 \pm 0.12$ & $0.012 \pm 0.002$ & $0.019 \pm 0.001$ & $0.30 \pm 0.09$ \\
\hline
\end{tabular}

Data are mean \pm SEM.

$$
\frac{m_{1}}{m}=\frac{V_{o} c_{a}}{m}=\frac{V_{o}}{V}
$$

Eq. 8

where $V$ is an apparent volume of distribution $\left(\mathrm{mL} / \mathrm{cm}^{3}\right)$ that equals the ratio of the total quantity of tracer in the tissue and the arterial concentration of the tracer as a function of time. With a vascular volume close to $3 \%$ in brain tissue, the volume $V$, equal to the ratio $m / c_{a}$, cannot exceed more than $3 \mathrm{~mL} \mathrm{~cm}^{-3}$ for the vascular tracer in brain to be less than $1 \%$ of the total radioactivity in the brain. After that time, the transfer of ${ }^{11} \mathrm{C}$-yohimbine to the tissue from the vascular compartment can be described with a single-compartment equation that replaces the 2-compartment equation for times great enough to render the radioactivity in the vascular volume negligible in relation to the total radioactivity in the tissue, which then obeys the equation,

$$
m(T)=K_{1} \int_{0}^{T} C_{a}(t) d t-k_{2}^{\prime} \int_{0}^{T} m(t) d t .
$$

Eq. 9

Rearrangement of this basic equation yields linear plots with positive or negative slopes. Here, we compare 6 different linear plots that estimate the kinetic parameters that govern uptake and distribution of ${ }^{11} \mathrm{C}$-yohimbine in the brain (14-16).
The kinetic variables calculated from the radioactivity records include the apparent volume of distribution as a function of the duration of circulation of the tracer (in units of $\mathrm{mL} \mathrm{cm}^{-3}$ ),

$$
V_{a p p}(T) \equiv \frac{\int_{0}^{T} m(t) d t}{\int_{0}^{T} C_{a}(t) d t}
$$

as well as the apparent net clearance of the tracer from plasma to brain as a function of circulation time (in units of $\mathrm{mL} / \mathrm{cm}^{3} / \mathrm{min}$ ),

$$
K_{a p p}(T) \equiv \frac{m(T)}{\int_{0}^{T} C_{a}(t) d t},
$$

Eq. 11

and the apparent residence time in the tissue (in units of minutes),

$$
\Theta(T) \equiv \frac{\int_{0}^{T} m(t) d t}{m(T)},
$$

Eq. 12

such that $V_{\text {app }}(T)=K_{\text {app }}(T) \Theta(T)$. When the primary variables are combined, they define 2 plots of negative slope (N1 and N2), or 4 plots of positive slope (P1-P4). In the "clearance plot" (N1), the ordinate intercept is the apparent clearance, $K_{\text {app }}(T)$, of tracer from plasma to 
brain, and the slope is $-k_{2}^{\prime}$, such that the abscissa intercept is the estimate of $V_{T}(17,18)$,

$$
\text { (N1) } \quad K_{\text {app }}(T)=K_{1}-k_{2}^{\prime} V_{a p p}(T)=k_{2}^{\prime}\left(V_{T}-V_{a p p}(T)\right), \quad \text { Eq. } 13
$$

whereas in the reverse "reciprocal ratio plot" (N2) (19), $V_{a p p}(T)$ is plotted against $K_{\text {app }}(T)$, such that the ordinate intercept is the estimate of the $V_{T}$,

$$
\text { (N2) } \quad V_{a p p}(T)=V_{T}-\left(\frac{1}{k_{2}^{\prime}}\right) K_{\mathrm{app}}(T) .
$$

The first of the positive slope plots is the "ratio plot" (P1) (17), where the inverse of the unidirectional clearance $K_{1}$ is the ordinate intercept and the slope is the inverse of $V_{T}$,

$$
\text { (P1) } \frac{1}{K_{\text {app }}(T)}=\frac{\Theta(T)}{V_{T}}+\frac{1}{K_{1}},
$$

such that the second of the positive slope plots, the Logan plot (P2), is obtained by reversing the equation, as originally published by Logan et al. (20),

$$
\text { (P2) } \Theta(\mathrm{T})=V_{T}\left[\frac{1}{K_{\text {app }}(T)}\right]-\frac{1}{k_{2}^{\prime}},
$$

where $\Theta(T)$ is plotted against the inverse of clearance, such that the positive slope represents $V_{T}$. The plot becomes linear when the system has reached steady state.

The third positive slope, or "reciprocal time," plot originally was derived by Reith et al. (21), who plotted the reciprocal of $\Theta(\mathrm{T})$ against the reciprocal of $V_{a p p}(T)$,

$$
\text { (P3) } \frac{1}{\Theta(\mathrm{T})}=K_{1}\left[\frac{1}{V_{a p p}(T)}\right]-k_{2}^{\prime} \text {, }
$$

where the steady-state volume is obtained from the slope and the ordinate intercept. In the last of the 4 positive slope plots, the axes of P3 have been reversed,

$$
\text { (P4) } \frac{1}{V_{a p p}(T)}=\left(\frac{1}{K_{1}}\right)\left[\frac{1}{\Theta(\mathrm{T})}\right]+\frac{1}{V_{T}},
$$

where $V_{T}$ is the reciprocal of the ordinate intercept. We calculated $B P_{N D}$ as the ratio of the volume $V_{T}$ in ROIs with specifically displaceable binding and the volume $V_{N D}$ of distribution in a chosen reference region with specifically nondisplaceable binding, here selected as the volume determined for the corpus callosum $\left(B P_{N D}=0\right)(22)$.

$$
B P_{N D}=\frac{V_{T}}{V_{N D}}-1
$$

\section{Data Analysis}

Analyses were performed in GraphPad Prism 6.0 and results are reported as mean ( \pm SEM). The correlation of estimates of $V_{T}$ between the different linear models was tested by linear regression of frontal cortex $V_{T}$ determined with $\mathrm{P} 4$ versus the other models.

\section{RESULTS}

\section{Brain Time-Activity Curves and Metabolites}

The tracer readily entered brain tissue, as revealed by the standardized uptake value curves and the estimates of the unidirectional blood-brain clearances $\left(K_{1}\right)$ of the tracer. Standardized uptake value curves for all 27 VOIs of one subject are shown in Figure 1A. We used the fraction of radioactivity in plasma, relative to the radioactivity in a given VOI, as shown in Figure $1 \mathrm{~B}$, to determine the time after which the fraction would be negligible and the plots henceforth linear, exemplified by frontal cortex and hippocampus, where, on average, the vascular tracer amounted to less than $6 \%$ after $12.5 \mathrm{~min}$. The fraction of intact tracer declined in plasma as a function of time. When we genotyped the CYP mixed-function oxidase CYP2D6 in all subjects, we classified 2 subjects as fast metabolizers and 3 subjects as moderate metabolizers. We classified one subject with no functional CYP2D6 genetic variants as a nonmetabolizer as shown in Table 1 and Figure 2.

Blood-Brain Clearances, Efflux Rates, Volumes of Distribution, and Binding Potentials of ${ }^{11} \mathrm{C}$-Yohimbine

After the time of negligible tracer in the vascular space (12.5 $\mathrm{min}$ ), the N1-P4 plots based on unmetabolized tracer in plasma assumed linearity and yielded comparable estimates of the 3 parameters $K_{1}, V_{T}$, and $k_{2}^{\prime}$ as shown in Figure 3. In line with this observation, we noted a highly significant correlation between

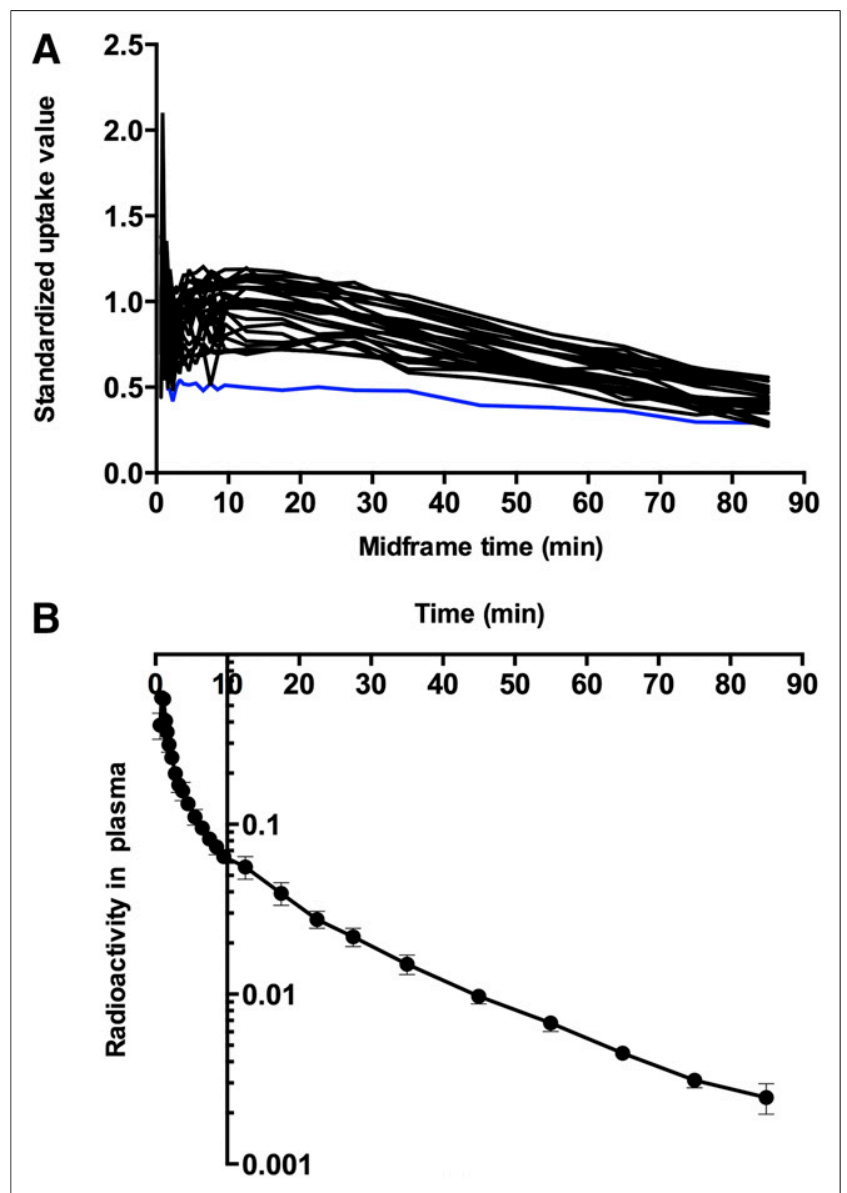

FIGURE 1. (A) Examples of standardized uptake value curves for all 27 VOls of ${ }^{11} \mathrm{C}$-yohimbine presented for subject 5 (abscissa is mid-frame time [minutes] and ordinate is standardized uptake value of radioactivity in VOIs). Blue line shows corpus callosum, which was used as reference region for calculation of binding potentials $\left(B P_{N D}\right)$. (B) Radioactivity in plasma, expressed as fraction of radioactivity in VOls, is shown on ordinate, and abscissa represents time (minutes). 


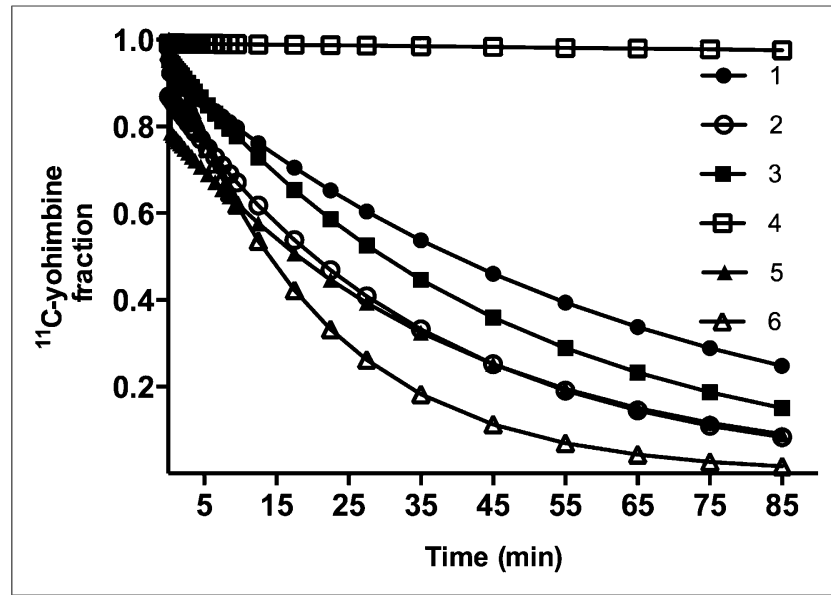

FIGURE 2. Fractions of unchanged ${ }^{11} \mathrm{C}$-yohimbine were interpolated to match time points of tissue time-activity curves, presented here for all 6 subjects. Ordinate represents intact ${ }^{11} \mathrm{C}$-yohimbine, and abscissa shows time (minutes).

estimates of $V_{T}$ in the frontal cortex determined with the plot P4 method and the remaining plots with $P<0.0001$ and mean $R^{2}=$ 0.9974 ( \pm 0.0007$)$. As the estimates were comparable, only $V_{T}$ and $K_{1}$ values from plot $\mathrm{P} 4$ and the efflux rate constant $k_{2}^{\prime}$ from plot $\mathrm{N} 2$ are presented here. We calculated the $B P_{N D}$ values listed in Table 2 for selected VOIs from the ratios of $V_{T}$ estimates to the estimate in the corpus callosum. The mean values of $V_{T}$ ranged from 0.82 $( \pm 0.16) \mathrm{mL} \mathrm{cm}^{-3}$ in the right frontal cortex to $0.46( \pm 0.09) \mathrm{mL}$ $\mathrm{cm}^{-3}$ in the corpus callosum, as also listed in Table 2. Estimates of $K_{1}$ and $k_{2}^{\prime}$ are listed in Table 2 for selected VOIs.

The estimates of $K_{1}$ and $k_{2}^{\prime}$ ranged from $0.020( \pm 0.003) \mathrm{mL}$ $\mathrm{cm}^{-3} \mathrm{~min}^{-1}$ and $0.023( \pm 0.001) \mathrm{min}^{-1}$, respectively, in the right frontal cortex, to $0.009( \pm 0.001) \mathrm{mL} \mathrm{cm}^{-3} \mathrm{~min}^{-1}$ and 0.019 ( \pm 0.003$) \mathrm{min}^{-1}$, respectively, in the corpus callosum. The estimates of $K_{1}$ are consistent with blood-brain extraction fractions of $5 \%$. A parametric image of the average estimates of $V_{T}$ in 5 subjects obtained with the plot P2 (Logan plot) is shown in Figure 4. In subject 6 , the parametric image was of unacceptably poor quality because of a combination of minimum dose of radioactivity and maximum rate of metabolism in plasma. Mean values of $B P_{N D}$, calculated and averaged from estimates of $V_{T}$ obtained from regions including the corpus callosum as reference by means of plot $\mathrm{P} 4$, are listed in Table 2, where they range from 0.6 to 0.8 in the cortex and from 0.2 to 0.5 in subcortical regions.

\section{DISCUSSION}

Animal studies from this laboratory have suggested that ${ }^{11} \mathrm{C}$ yohimbine is a selective and displaceable $\alpha_{2}$-adrenoceptor antagonist when used in tracer concentrations $(11,12)$. To measure accumulation of the tracer in human brain, we determined the uptake and binding of ${ }^{11} \mathrm{C}$-yohimbine in 5 healthy male volunteers (subject 6
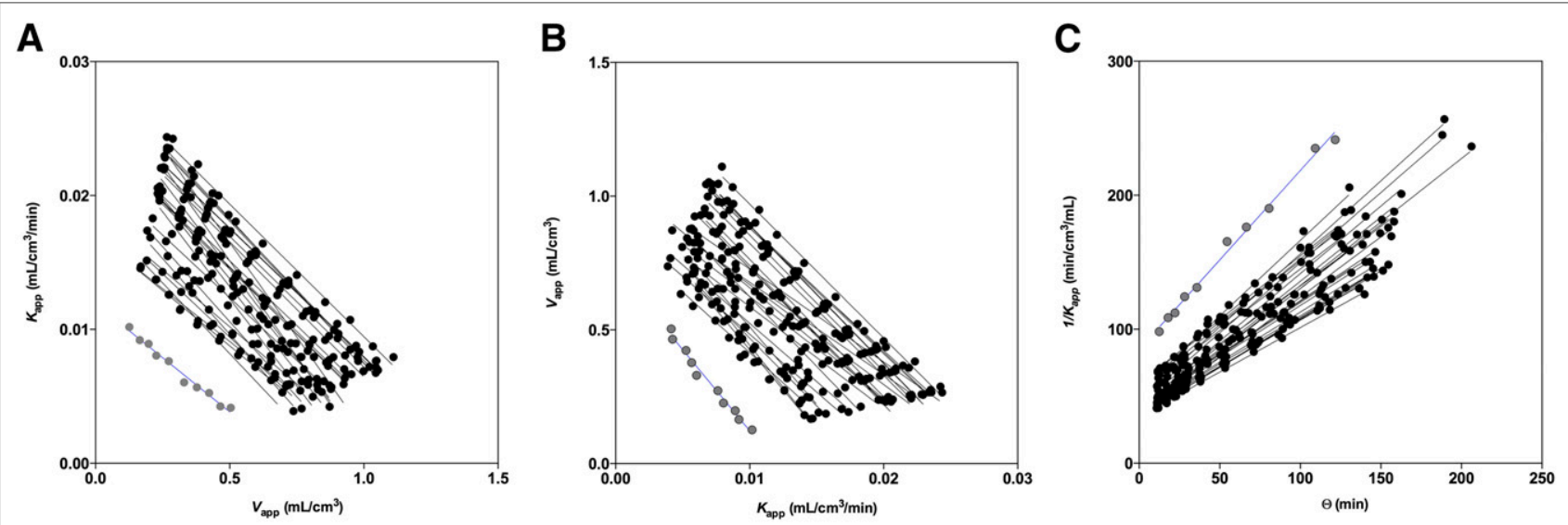

D

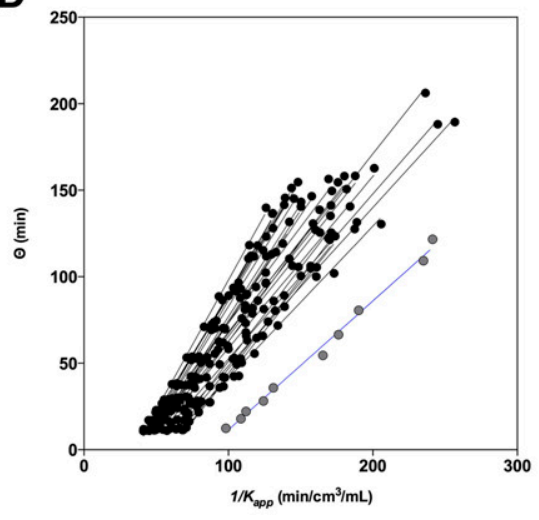

E

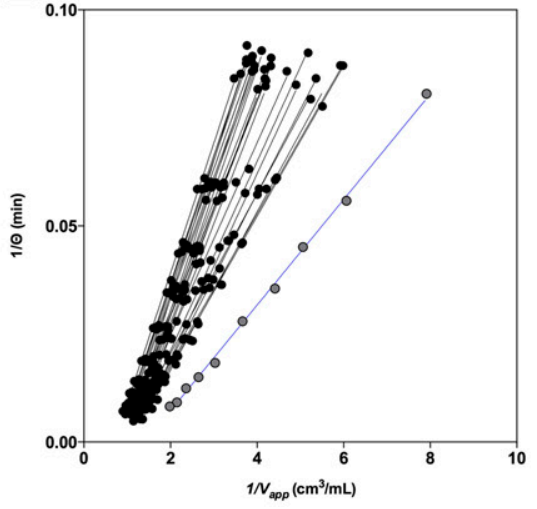

$\mathbf{F}$

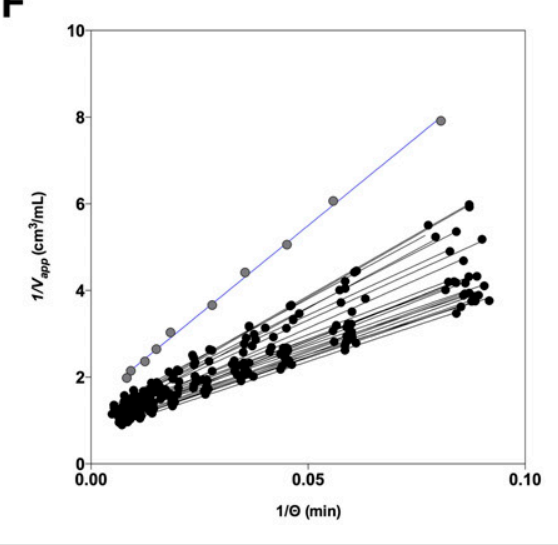

FIGURE 3. Six linearized solutions were used to derive linear regression estimates of kinetic parameters, using metabolite-corrected plasma curves as input function: plot N1 (A), plot N2 (B), plot P1 (C), plot P2 (D), plot P3 (E), plot P4 (F). Blue lines represent corpus callosum. 


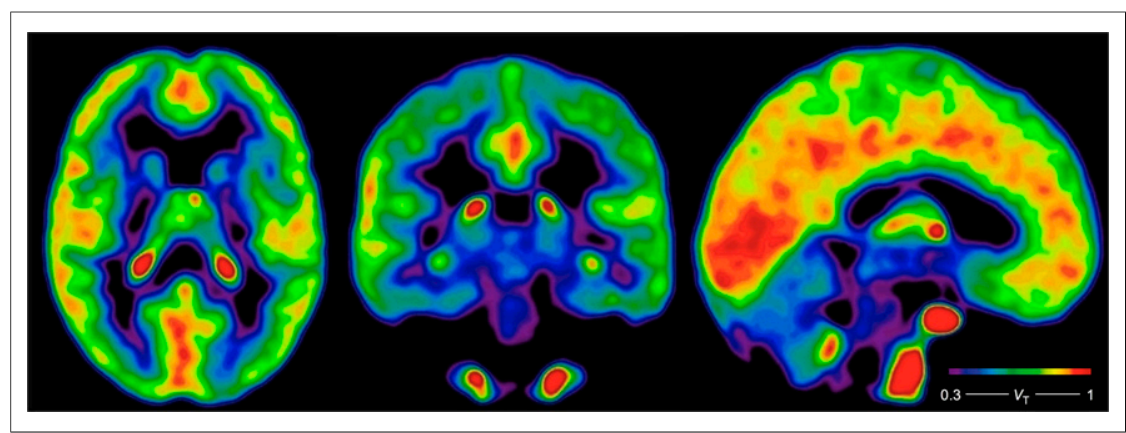

FIGURE 4. Average parametric images of voxelwise $V_{T}$ estimates in 5 of 6 subjects, estimated with P2 plot (Logan plot). Color bar gives estimates of $V_{T}$ in units of $\mathrm{mL} \mathrm{cm}^{-3}$. of $\alpha_{2}$ adrenoceptors, which may complicate the choice of thalamus as a reference region (6). In vitro studies have shown that white matter and the corpus callosum have negligible $\alpha_{2}$-adrenoceptor binding sites and thus may be considered for use as a reference region. The tentative choice of the corpus callosum as a reference region here was based on the finding of the lowest $V_{T}$, as estimated with the plot P4. The high selectivity of the PET ligand, ${ }^{11} \mathrm{C}$-yohimbine, for $\alpha_{2}$-adrenoceptors (11), with the highest $V_{T}$ values being in regions with known high density of $\alpha_{2}$ adrenoceptors, indicates that this ligand is useful for in vivo imaging of $\alpha_{2}$ adrenoceptors in humans.

was excluded). The results revealed about 5\% extraction of the tracer, sufficient for determination of regionally averaged uptake in 5 volunteers, and similarly acceptable for voxel-based mapping in only 5 of the 6 volunteers.

Linearized solutions of the underlying differential equations such as the Logan plot (P2) were used here to graphically estimate the kinetic parameters of ${ }^{11} \mathrm{C}$-yohimbine (17-21). We analyzed the present data with 6 different linearized solutions, including the Logan plot, to identify the plot most suitable for future applications of ${ }^{11} \mathrm{C}$-yohimbine. All solutions, here abbreviated N1-P4, of which P2 is the Logan plot, adequately fitted the data after the plots assumed linearity approximately $12.5 \mathrm{~min}$ after administration of the bolus of radioligand as shown in Figure 3. The estimates of ${ }^{11} \mathrm{C}$-yohimbine $V_{T}$ in the frontal cortex determined with plot $\mathrm{P} 4$ significantly correlated with the 5 other linearizations $(P<$ $0.0001)$. The estimates ranged from $0.82 \mathrm{~mL} \mathrm{~cm}^{-3}$ in the right frontal cortex to $0.72 \mathrm{~mL} \mathrm{~cm}^{-3}$ in the left insula and $0.77 \mathrm{~mL}$ $\mathrm{cm}^{-3}$ in the left hippocampus. Subcortical structures, including the thalamus, caudate nucleus, putamen, and amygdala, had lower estimates of $V_{T}$. Surprisingly, white matter, excluding the corpus callosum, also had a moderate volume of distribution of ${ }^{11} \mathrm{C}$ yohimbine $\left(0.59 \mathrm{~mL} \mathrm{~cm}^{-3}\right)$, which may reflect the influence of partial volume.

Obtained from the linearized plots, the estimates of unidirectional blood-brain clearance of ${ }^{11} \mathrm{C}$-yohimbine, $K_{1}$, had a 2 -fold range whereas the rate of washout of the tracer had a narrow range from the right frontal cortex to the corpus callosum (Table 2). In the study by Hume et al. (23), the $K_{1}$ value in the frontal cortex of another PET ligand for $\alpha_{2}$ adrenoceptors, ${ }^{11} \mathrm{C}-\mathrm{RS}-15385-197$, was $0.009 \mathrm{~mL} / \mathrm{cm}^{3} / \mathrm{min}$, which is close to $K_{1}$ in the corpus callosum in the current study. In contrast, the tracer ${ }^{11} \mathrm{C}$-mirtazapine had a $K_{1}$ value approximately 10 times higher than that for ${ }^{11} \mathrm{C}$-yohimbine. However, ${ }^{11} \mathrm{C}$-mirtazapine lacks selectivity for $\alpha_{2}$ adrenoceptors (9).

Although the distribution volumes of ${ }^{11} \mathrm{C}$-yohimbine are modest compared with other PET radioligands and with ${ }^{11} \mathrm{C}$-mirtazapine, its binding potential is comparable to other noradrenergic tracers in successful use. For example, the binding potential of $(S, S)-{ }^{11} \mathrm{C}$ methylreboxetine, an antagonist of noradrenaline transporters, is 0.52 in the thalamus, which receives noradrenergic innervation (24).

Here, we calculated binding potentials as estimates of receptor availability as the ratio of $V_{T}$ estimated in VOIs with assumed displaceable binding, compared with a chosen reference region, corpus callosum. Hume et al. (23) used the thalamus as a reference region based on the assumption that it contains few $\alpha_{2}$-adrenoceptor binding sites. However, some thalamic nuclei show dense expression
The CYP-450 system and specifically the CYP2D6 enzyme in the liver are involved in the metabolism of yohimbine in the circulation, yielding 2 metabolites, 10-OH-yohimbine and 11-OHyohimbine (25). We genotyped the CYP2D6 enzyme and classified subjects as fast, moderate, or low metabolizers, depending on the number of functional CYP2D6 variants, with one subject classified as a nonmetabolizer. As expected, the fraction of unchanged ${ }^{11} \mathrm{C}$-yohimbine remained close to unity in this subject, whereas moderate and fast metabolizers had substantial breakdown of ${ }^{11} \mathrm{C}$ yohimbine. The results are consistent with an earlier study of yohimbine metabolism (25), which also identified one subject as a nonmetabolizer. Yohimbine metabolites may have some action at $\alpha_{2}$ adrenoceptors, albeit with lower affinity when compared with native yohimbine (26). Labeled 11-OH-yohimbine could hypothetically contribute to radioactivity in the brain, as samples of cerebrospinal fluid in previous studies have revealed transport across the blood-brain barrier (27). We plan to study radioactively labeled metabolites of yohimbine to estimate the degree to which they cross the blood-brain barrier and bind to targets in the brain.

The $\alpha_{2}$ adrenoceptors play important roles in the regulation of noradrenergic neurotransmission and are implicated in the pathology of neurodegenerative and neuropsychiatric disorders (27). It is likely, therefore, that in vivo imaging of $\alpha_{2}$ adrenoceptors with ${ }^{11} \mathrm{C}$-yohimbine will provide insights into the underlying mechanisms of these disorders.

\section{CONCLUSION}

Here, we present ${ }^{11} \mathrm{C}$-yohimbine as a PET radioligand for vivo imaging of $\alpha_{2}$ adrenoceptors in humans. The relative distribution of ${ }^{11} \mathrm{C}$-yohimbine is similar to that predicted from in vitro studies, with the highest binding being in the cortex and hippocampus, and the binding suggests that ${ }^{11} \mathrm{C}$-yohimbine may be a suitable ligand for future studies of the role of $\alpha_{2}$ adrenoceptors in neurodegenerative and neuropsychiatric disorders of the human brain.

\section{DISCLOSURE}

The costs of publication of this article were defrayed in part by the payment of page charges. Therefore, and solely to indicate this fact, this article is hereby marked "advertisement" in accordance with 18 USC section 1734. This project received financial support from the Lundbeck Foundation (grant 6970) and from the Danish Council for Independent Research, Medical Sciences (grant 0602-02700). No other potential conflict of interest relevant to this article was reported. 


\section{REFERENCES}

1. Starke K. Presynaptic autoreceptors in the third decade: focus on $\alpha_{2}$-adrenoceptors. J Neurochem. 2001;78:685-693.

2. Weinshank RL, Zgombick JM, Macchi M, et al. Cloning, expression, and pharmacological characterization of a human alpha 2B-adrenergic receptor. Mol Pharmacol. 1990;38:681-688.

3. Kobilka BK, Matsui H, Kobilka TS, et al. Cloning, sequencing, and expression of the gene coding for the human platelet alpha 2-adrenergic receptor. Science. 1987;238:650-656.

4. Lomasney JW, Lorenz W, Allen LF, et al. Expansion of the alpha 2-adrenergic receptor family: cloning and characterization of a human alpha 2-adrenergic receptor subtype, the gene for which is located on chromosome 2. Proc Natl Acad Sci USA. 1990;87:5094-5098.

5. Bylund DB. Subtypes of alpha 1- and alpha 2-adrenergic receptors. FASEB J. 1992;6:832-839.

6. Pascual J, del Arco C, González AM, Pazos A. Quantitative light microscopic autoradiographic localization of $\alpha 2$-adrenoceptors in the human brain. Brain Res. 1992;585:116-127.

7. Perälä $\mathrm{M}$, Hirvonen $\mathrm{H}$, Kalimo $\mathrm{H}$. Differential expression of two $\alpha 2$-adrenergic receptor subtype mRNAs in human tissues. Brain Res Mol Brain Res. 1992;16:57-63.

8. Marien MR, Colpaert FC, Rosenquist AC. Noradrenergic mechanisms in neurodegenerative diseases: a theory. Brain Res Brain Res Rev. 2004;45:38-78.

9. Smith DF, Dyve S, Minuzzi L, et al. Inhibition of $\left[{ }^{11} \mathrm{C}\right]$ mirtazapine binding by alpha2-adrenoceptor antagonists studied by positron emission tomography in living porcine brain. Synapse. 2006;59:463-471.

10. Van der Mey M, Windhorst AD, Klok RP, et al. Synthesis and biodistribution of $\left[{ }^{11} \mathrm{C}\right] \mathrm{R} 107474$, a new radiolabeled alpha2-adrenoceptor antagonist. Bioorg Med Chem. 2006; 14:4526-4534.

11. Jakobsen S, Pedersen K, Smith DF, Jensen SB, Munk OL, Cumming P. Detection of alpha2-adrenergic receptors in brain of living pig with $\left[{ }^{11} \mathrm{C}\right]$ yohimbine. $\mathrm{J} \mathrm{Nucl}$ Med. 2006;47:2008-2015.

12. Phan JA, Jakobsen S, Simonsen M, Nahimi A, Landau AM, Gjedde A. Amphetamineinduced inhibition of [C-11]yohimbine binding in rat brain. J Cereb Blood Flow Metab. 2012;32(suppl):S98-S99.

13. Hammers A, Koepp MJ, Free SL, et al. Implementation and application of a brain template for multiple volumes of interest. Hum Brain Mapp. 2002;15:165-174.

14. Innis RB, Cunningham VJ, Delforge J, et al. Consensus nomenclature for in vivo imaging of reversibly binding radioligands. J Cereb Blood Flow Metab. 2007; 27:1533-1539.
15. Gjedde A, Wong DF, Rosa-Neto P, Cumming P. Mapping neuroreceptors at work: on the definition and interpretation of binding potentials after 20 years of progress. Int Rev Neurobiol. 2005;63:1-20.

16. Gjedde A, Bauer WR, Wong DF. Neurokinetics: The Dynamics of Neurobiology in Vivo. New York, NY: Springer; 2010:103-126.

17. Gjedde A. Calculation of cerebral glucose phosphorylation from brain uptake of glucose analogs in vivo: a re-examination. Brain Res. 1982;257:237-274.

18. Cumming P, Léger GC, Kuwabara H, Gjedde A. Pharmacokinetics of plasma $6-\left[{ }^{18} \mathrm{~F}\right]$ fluoro-L-3,4-dihydroxyphenylalanine $\left[{ }^{18} \mathrm{~F}\right] \mathrm{FDOPA}$ in humans. J Cereb Blood Flow Metab. 1993;13:668-675.

19. Gjedde A, Gee AD, Smith DF. Basic CNS drug transport and binding kinetics in vivo. In: Begley DJ, Bradbury MW, Kreuter J, eds. The Blood-Brain Barrier and Drug Delivery to the CNS. New York, NY: Marcel Dekker; 2000:225-242.

20. Logan J, Fowler JS, Volkow ND, et al. Graphical analysis of reversible radioligand binding from time-activity measurements applied to $\left[\mathrm{N}^{11} \mathrm{C}\right.$-methyl](-)-cocaine PET studies in human subjects. J Cereb Blood Flow Metab. 1990;10:740-747.

21. Reith J, Dyve S, Kuwabara H, Guttman M, Diksic M, Gjedde A. Blood-brain transfer and metabolism of $6-\left[{ }^{18} \mathrm{~F}\right]$ fluoro-L-DOPA in rat. J Cereb Blood Flow Metab. 1990;10:707-719.

22. Gjedde A, Kumakura Y, Cumming P, Linnet J, Møller A. Inverted-U-shaped correlation between dopamine receptor availability in striatum and sensation seeking. Proc Natl Acad Sci USA. 2010;107:3870-3875.

23. Hume SP, Hirani E, Opacka-Juffry J, et al. Evaluation of [O-methyl- $\left.{ }^{11} \mathrm{C}\right] \mathrm{RS}-$ 15385-197 as a positron emission tomography radioligand for central a2-adrenoceptors. Eur J Nucl Med. 2000;27:475-484.

24. Hannestad J, Gallezot JD, Planeta-Wilson B, et al. Clinically relevant doses of methylphenidate significantly occupy norepinephrine transporters in humans in vivo. Biol Psychiatry. 2010;68:854-860.

25. Le Corre P, Dollo G, Chevanne F, Le Verge R. Biopharmaceutics and metabolism of yohimbine in humans. Eur J Pharm Sci. 1999;9:79-84.

26. Berlan M, Verge RL, Galitzky J, Corre PL. $\alpha 2$-adrenoceptor antagonist potencies of two hydroxylated metabolites of yohimbine. Br J Pharmacol. 1993;108:927932.

27. Le Corre PA, Peskind ER, Chevanne F, Raskind MA, Le Verge R. Cerebrospinal fluid and plasma disposition of yohimbine and 11-hydroxy-yohimbine in young and older healthy subjects, and Alzheimer's disease patients. Eur J Clin Pharmacol. 1997;52:135-138. 